\title{
Multiplexed holographic data page storage on a polyvinyl alcohol/acrylamide photopolymer memory
}

\author{
Elena Fernández, ${ }^{1, \star}$ Manuel Ortuño, ${ }^{2}$ Sergi Gallego, ${ }^{2}$ Andrés Márquez, ${ }^{2}$ Celia García, ${ }^{1}$ \\ Augusto Beléndez, ${ }^{2}$ and Inmaculada Pascual ${ }^{1}$ \\ ${ }^{1}$ Departamento de Óptica, Farmacología y Anatomía, Universidad de Alicante, Apartado 99, E-03080 Alicante, Spain \\ ${ }^{2}$ Departamento de Física, Ingeniería de Sistemas y Teoría de la Señal, Universidad de Alicante, \\ Apartado 99, E-03080 Alicante, Spain \\ *Corresponding author: elena.fernandez@ua.es
}

Received 20 March 2008; accepted 12 July 2008;

posted 25 July 2008 (Doc. ID 93977); published 21 August 2008

\begin{abstract}
Holographic data pages were multiplexed in different thickness layers of a polyvinyl alcohol/acrylamide photopolymer. This material is formed of acrylamide photopolymers, which are considered interesting materials for recording holographic memories. A liquid crystal device was used to modify the object beam and store the data pages. A peristrophic multiplexing method is used to store a large number of data pages in the same spot in the material. The bit error rate was calculated fitting the histograms of the images to determine what parameters improve the quality of the images. (C) 2008 Optical Society of America
\end{abstract}

OCIS codes: $\quad 090.0090,090.2900,090.2870,100.2960,210.0210,210.4810$.

\section{Introduction}

Due to the importance acquired by new technologies (computers and Internet), the demand for more capacity, more density, and faster readout rates has increased considerably. The conventional optical memory technologies, like CD-ROMs and DVDs, are two-dimensional surface-storage techniques, and so have almost arrived at the limit of their capacity. For this reason, in recent years a lot of attention has been centered on three-dimensional (3D) holographic disks and memories [1-4]. Recently, many studies have been focused on the characterization and optimization of thick holographic recording materials $[\underline{5}, 6]$ in order to obtain the maximum data storage capacity. Some companies such as Aprilis [7] or InPhase $[8,9]$ have already created the first prototypes of holographic optical storage systems capable of storing from 200 Gbytes to 1.6 Tbytes.

0003-6935/08/254448-09\$15.00/0

(C) 2008 Optical Society of America
Since photopolymers have excellent holographic characteristics, such as high refractive index modulation $[10,11]$, large dynamic range $[2,12-14]$, good light sensitivity, real time image development, high optical quality, and low cost, they have been used as the base of new 3D holographic disks. In addition to this, their properties such as energetic sensitivity or spectral sensitivity can be easily changed by modifying their composition $[10,14-16]$.

In this study, we focus on the optimization of a holographic memory setup multiplexing a large number of data pages using a liquid crystal display (LCD) and a polyvinyl alcohol (PVA)/acrylamide photopolymer.

Twisted-nematic liquid crystal displays (TNLCDs) have been extensively studied in recent years for application to spatial light modulators (SLMs) used in many applications in optics to modify in real time the amplitude or phase of a light beam [17-23]. This LCD can be used to design programmable optical elements, such as lenses and data pages or in holographic data storage. In particular in holographic 
data storage, LCDs allow data pages to be recorded in real time in the holographic recording material.

The photopolymer used in this work was composed of acrylamide (AA) as the polymerizable monomer, triethanolamine (TEA) as radical generator, $\mathrm{N}, \mathrm{N}^{\prime}$ methylene-bis-acrylamide (BMA) as crosslinker, yellowish eosin (YE) as sensitizer, and a binder of polyvinyl alcohol (PVA) [24]. Layers about $80 \pm 10 \mu \mathrm{m}$ [25], $250 \pm 10 \mu \mathrm{m}$, and $\overline{50} 0 \pm 10 \mu \mathrm{m}$ were used $[15,16]$.

Different objects with white and black pixels were used to simulate data page bits (ones and zeros). During the storage process, these data pages are stored in the photopolymer. When the hologram has been stored, it is illuminated in the reconstruction stage by the same plane wave as in the recording process in order to prevent the appearance of aberrations in the reconstructed image. Using an optical system, the stored information is imaged onto a Cohu 4710 Series Monochrome CCD camera connected to a personal computer, where the images are analyzed and processed.

These objects were multiplexed in the three different thicknesses layers. To maximize full dynamic range of the material $[2,12,13]$ and to store a large number of holograms, exposure was gradually increasing while the holograms were being stored.

Once the images have been obtained, a criterion has to be used to assess the quality of the different images and to compare them with the original object. In order to evaluate the image quality, its histogram is used to calculate the bit error rate (BER) [26-29] and to determine the contrast between white and black pixels. BER values of each image are calculated to decide what parameters provide the best image quality (greater contrast and less noise).

\section{Experimental Setup}

\section{A. Preparation of the Material}

The holograms are recorded in a photopolymer composed of acrylamide (AA) as the polymerizable monomer, triethanolamine (TEA) as radical generator, $\mathrm{N}, \mathrm{N}^{\prime}$ methylene-bis-acrylamide (BMA) as crosslinker, yellowish eosin (YE) as sensitizer, and a binder of polyvinyl alcohol (PVA). Introduction of BMA in the composition improves the energetic sensitivity and diffraction efficiency of the material and, in addition, gives a greater stability to the stored grating, thereby preventing it from disappearing with time.

Table 1 shows the component concentrations of the photopolymer compositions used to obtain layers about $80 \mu \mathrm{m}$ thick with the composition 1, layers about $250 \mu \mathrm{m}$ with the composition 2, and layers about $500 \mu \mathrm{m}$ with the composition 3 . In previous papers the material was optimized in order to obtain a greater diffraction efficiency [15,16,25].

A solution of PVA in water forms the matrix, and this is used to prepare the mixture of AA, BMA, and a photopolymerization initiator system composed of TEA and YE. The mixture is made under red light,
Table 1. Concentrations of the Photopolymer Compositions

\section{Photopolymer} Acrylamide

Triethanolamine

Yellowish eosin

$\mathrm{N}, \mathrm{N}^{\prime}$ methylene-bisacrylamide
Polyvinyl alcohol

\section{Composition Composition Composition}

$6.6 \% \mathrm{~W} / \mathrm{V} \quad 13.4 \% \mathrm{~W} / \mathrm{V} \quad 13.3 \% \mathrm{~W} / \mathrm{V}$

$\begin{array}{lll}0.33 \mathrm{M} & 0.34 \mathrm{M} & 0.35 \mathrm{M}\end{array}$

$0.17 \mathrm{M} \quad 0.15 \mathrm{M} \quad 0.15 \mathrm{M}$

$2.4 \times 10^{-4} \mathrm{M} 1.4 \times 10^{-4} \mathrm{M} 0.9 \times 10^{-4} \mathrm{M}$

$0.027 \mathrm{M} \quad 0.037 \mathrm{M} \quad 0.04 \mathrm{M}$ deposited by gravity on a glass plate, and left in the dark for one day to allow the water to evaporate in conditions of temperature $T=22{ }^{\circ} \mathrm{C}$ and relative humidity $\mathrm{RH}=55 \%$. These conditions of drying time, temperature, and relative humidity are optimized to obtain the maximum diffraction efficiency of the gratings. Once dry, the glass is cut into squares of $5 \mathrm{~cm} \times 5 \mathrm{~cm}$.

\section{B. Holographic Setup}

Holographic data pages were recorded using the setup shown in Fig. 1 . The light source was a diodepumped frequency-doubled $\mathrm{Nd}: \mathrm{YVO}_{4}$ laser (Coherent Verdi V2) with a wavelength of $532 \mathrm{~nm}$. The polarized beam was split into two beams with a beam splitter. Then the beams were spatially filtered using a $40 \times$ objective lens and a $10 \mu \mathrm{m}$ pinhole. The filtered beams were collimated using the lenses L1 and L2 and the diaphragms D1 and D2. The diameter of these beams was $1.5 \mathrm{~cm}$, obtained with D3 and D4, and the sum of the intensities of the two beams was $3 \mathrm{~mW} / \mathrm{cm}^{2}$. The incident angles of the object beam and the reference beam at the recording medium were $17.4^{\circ}$ (measured in air). In previous studies in which diffraction gratings were stored, this angle was used to obtain a spatial frequency of 1125 lines $/ \mathrm{mm}$ $[12,13]$, and the material's behavior was seen to be good. For this reason, the same angle between the beams was used in this study.

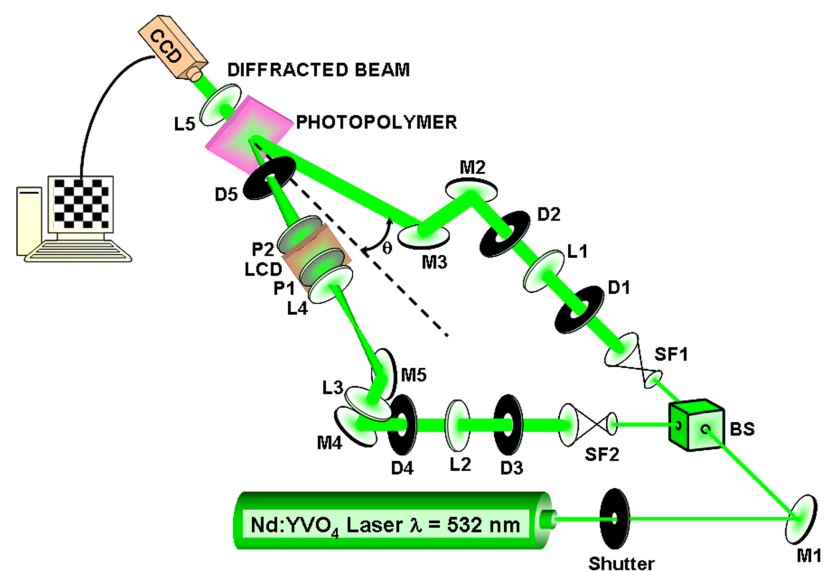

Fig. 1. (Color online) (Color online) Experimental setup: BS, beam splitter; $\mathrm{Mi}$, mirror; Li, lens; Di, diaphragm; SFi, microscope objective lens and pinhole; SLM, spatial light modulator; Pi, polarizer; CCD, charge coupled device. 
One of the beams was the object beam, and the other was the reference beam. The object beam was directed onto the surface of a $800 \times 600 \mathrm{TN}$ LCD with a pixel pitch of $32 \mu \mathrm{m}$. The LCD was placed between two polarizers, one to each side of the LCD. The set of LCD and polarizers was used as a SLM. The lens L3 $\left(f^{\prime}=200 \mathrm{~mm}\right)$ was used to obtain a convergent beam, and the lenses L4 and L5 $\left(f^{\prime}=\right.$ $100 \mathrm{~mm}$ ) were placed to do the Fourier transform (FT) and the inverse Fourier transform (IFT) of the data pages. We used this scheme because we obtained a flexibility configuration: the size of the FT was under control by moving the LCD along the axis. By increasing $d$, the size of the FT was made larger, and decreasing $d$, the size of the FT was made smaller. A diaphragm was placed just before the photopolymer to block all the orders that leave the LCD except the central order. If the other orders were not blocked, they would also be stored in the materi$\mathrm{al}$, and during reconstruction, interference patterns would be observed on the image, thus worsening its quality. The other beam, the reference beam, was a plane wave that interfered with the object beam at the surface of the material. These beam intensities were measured at the position where the photopolymer must be placed when the holograms are stored.

In the reconstruction stage, the stored hologram that contained the information of the data page was illuminated with the reference beam, but at a very low intensity so as not to erase the hologram because the material was sensitive at this wavelength. Another lens was placed behind the photopolymer to do the inverse Fourier transform (IFT) of the diffracted beam on the surface of the charge coupled device (CCD). A computer sends the data pages to the LCD and captures images reconstructed by the CCD.

In order to ensure the stability of the experimental setup for long exposure times, a holographic table with an antivibration system was used.

\section{Results and Discussion}

In this paper, we want a large number of data pages be stored in a PVA/acrylamide photopolymer. For this reason, holograms have been multiplexed for different thicknesses of the material, $80 \mu \mathrm{m}, 250 \mu \mathrm{m}$, and $500 \mu \mathrm{m}$, using the compositions in Table 1 . In each layer, binary data pages with a random pixel form were stored. These objects have a different number of pixels: $300 \times 300,400 \times 400,500 \times 500$, and $800 \times$ 600 pixels. This allows us to study the behavior of the photopolymer material when data pages with different numbers of pixels are multiplexed.

These objects were stored in the same position in the material using peristrophic multiplexing because previous papers showed that when a not very large number of holograms (fewer than 200 holograms) are to be stored, a higher diffraction efficiency is obtained with peristrophic multiplexing [13]. By changing the thickness of the material, the number of holograms that can be stored will also change. With a greater thickness, the material will have a greater dynamic range and more holograms may be stored [2] .

The holograms were stored with an angular separation of $3^{\circ}$. The angular selectivity was measured for the different thicknesses used in this study. For a thickness of $500 \mu \mathrm{m}$, we obtained an angular selectivity of $1^{\circ}$, for $250 \mu \mathrm{m}, 1.5^{\circ}$, and for $80 \mu \mathrm{m}, 2^{\circ}$. Therefore an angular separation of $3^{\circ}$ is more than sufficient to prevent the holograms from overlapping.

The holograms were stored with a reference to object beam ratio of 100 and a reading beam intensity of $0.03 \mathrm{~mW} / \mathrm{cm}^{2}$. In a previous study on the influence of these parameters on the quality of the stored images [25], these values were found to give a greater image quality and therefore a lower BER.

\section{A. Optimization of the LCD}

As described in the holographic setup section, an LCD (LCD2002 Holoeye SONY LCX016AL-6 $800 \times 600$ pixels) was placed in the object beam to modify the wavefront and store this variation in the photopolymer. The variation may be in phase or amplitude. In this study, the wavefront amplitude is modified, while its phase is maintained constant, and this modification is the object to be stored in the photopolymer. The object has two states: white, with a maximum amplitude transmittance, and black, with a minimum amplitude transmittance. The optimization process of the LCD can be seen in [17-23].

LCDs change the polarization state of the incident light. This may mean that not all the light incident on the LCD is transmitted, since some of it is reflected or absorbed. Therefore it is advisable to place two polarizers, one before and one after the LCD, in order to transmit the maximum light possible. However, to achieve this, the LCD and polarizers must be calibrated correctly, which means that the angles at which the latter are to be placed in order to achieve maximum efficiency and minimum loss must be calculated. In our study, the objective was also to obtain the maximum contrast between transparent and opaque zones of the LCD with either no or minimal phase variation. Therefore before sending the objects to the LCD for storage in the photopolymer, the LCD must be correctly calibrated.

This is done using the method described in $[19,20]$, which consists of two calibration steps. In the first step, the LCD is turned off and no voltage is applied. In this step, three parameters that are independent of the voltage are calculated: the total twist angle $(\alpha)$, the orientation of the molecular director at the input face $\left(\psi_{D}\right)$, and the maximum birefringence $\left(\beta_{\max }\right)$. The parameters obtained with the LCD calibrated while turned off are shown in Table 2.

In the second step, the parameters dependent on voltage are measured. These are related to the variation in optical anisotropic properties throughout the thickness of the cell as a function of the voltage applied. The model attempts to take into account that the liquid crystal molecules near the glass are practically adhered to its surface and cannot reorientate 
Table 2. LCD Calibration Parameters Independent of the Voltage Applied

\begin{tabular}{ccccc}
\hline & \multicolumn{2}{c}{$\beta_{\max }$} & $\beta_{\max }$ & $\beta_{\max }$ \\
$\alpha$ & $\psi_{D}$ & $\left(\lambda_{0}=633 \mathrm{~nm}\right)$ & $\left(\lambda_{0}=532 \mathrm{~nm}\right)$ & $\left(\lambda_{0}=442 \mathrm{~nm}\right)$ \\
\hline $94^{\circ} \pm 1^{\circ}$ & $45^{\circ} \pm 1^{\circ}$ & $118^{\circ} \pm 1^{\circ}$ & $168^{\circ} \pm 1^{\circ}$ & $199^{\circ} \pm 1^{\circ}$ \\
\hline
\end{tabular}

themselves when the voltage is applied. Thus, the total thickness $d$ of the LCD may be decomposed into two lateral regions of width $d_{1}$ and a central region of width $d_{2}$. In this way, the anisotropic properties of the LCD may be modeled using two voltage-dependent parameters, birefringence $\beta$ and $\delta$, which are expressed in Eq. (1):

$$
\beta(V)=\pi \Delta n d_{2} / \lambda_{0}, \quad \delta(V)=\pi \Delta n_{\max } d_{1} / \lambda_{0},
$$

where $\lambda_{0}$ is the wavelength of the light and $\Delta n$ is the difference between the ordinary and extraordinary indices, with $\Delta n_{\max }$ being the maximum value.

From the curves of $\beta(V)$ and $\delta(V)$, we can find the angles at which the polarizers must be placed in the experimental setup to modulate the incident beam. In our study, our aim is to obtain the maximum contrast between transparent and opaque zones of the LCD with minimum variation in the phase. In this case, the angles found are $\varphi_{1}=296^{\circ}$ for the polarizer in front of the LCD and $\varphi_{2}=111^{\circ}$ for the polarizer behind it.

\section{B. Calculation of the Bit Error Rate}

After one of the objects has been stored in the photopolymer, the hologram formed is reconstructed by illuminating it with the reference beam. The diffractive beam obtained is imaged onto the CCD. If the holographic reconstruction were perfect, the images obtained would have uniform white and black pixels. However, many reasons exist that may distort the image (such as small imperfections in the material, particles that may be found in any element of the setup, or poor contrast of the image obtained due to insufficient exposure). Since the images obtained are not perfect and exhibit noise, it is necessary to measure a parameter that quantifies the image quality. This parameter is the bit error rate (BER).

The BER is defined as the probability of having erroneous bits in the image. To calculate the BER $[25,26]$, first, in Fig. 2, we represent in logarithmic scale the probability of obtaining a certain gray level in the black or the white regions. In Fig. 2, the probability in the black regions is represented by solid circles, and the probability in the white regions is represented by empty circles. As can be seen, the two probability distributions are clearly distinguishable, although there is a point at which they intersect. This point of intersection of the two distributions is called $x_{c}$.

Once the probability distributions have been obtained from the graph (Fig. 2), both distributions are fitted to a probability function. In this study, they are fitted to a Gaussian equation (Eq. (2)):

$$
W\left(x_{0}, \sigma ; x\right)=\frac{1}{\sqrt{2 \pi \sigma}} \exp \left(-\frac{\left(x-x_{0}\right)^{2}}{2 \sigma^{2}}\right)
$$

where $x$ represents each gray level in the image, $x_{0}$ is the point at which the Gaussian distribution is centered, and $\sigma$ is the width of the Gaussian distribution. The reason why they are fitted to a Gaussian equation is that it has been verified that most probability distributions obtained from an image captured by a CCD may be expected to follow this type of distribution.

Once the adjustments for the probability distribution of both white and black pixels have been made, the BER is calculated from Eq. (3):

$$
\mathrm{BER}=\frac{1}{2}\left[\int_{0}^{x_{c}} W_{W}(x) \mathrm{d} x+\int_{x_{c}}^{\infty} W_{B}(x) \mathrm{d} x\right]
$$

where $W_{W}$ are $W_{B}$ are the adjustments of the probability distribution of white and black pixels, respectively, and $x_{c}$ is the point of intersection of the two probability distributions.

Using this algorithm, the BERs of all the images are calculated and are discussed in the following subsections.

\section{Thickness of $80 \mu \mathrm{m}$}

As mentioned above, data pages were stored with four different pixel sizes. In this subsection, these four data pages are multiplexed in the $80 \mu \mathrm{m}$ thick material. Each of the four objects is stored at the same position in the material with an angular separation of $3^{\circ}$. In addition, they are stored with a beam ratio of 100 and reconstructed on the CCD with a reading beam intensity of $0.03 \mathrm{~mW} / \mathrm{cm}^{2}$. In previous studies it was found that when all the holograms were stored using the same exposure time, the last holograms were stored with lower diffraction efficiency or not even formed. In order to increase the diffraction efficiency of the last holograms, it was found necessary to increase the exposure of the holograms as they were being stored $[12,13,30]$. Therefore, since in this study the objective is to store as many data pages as

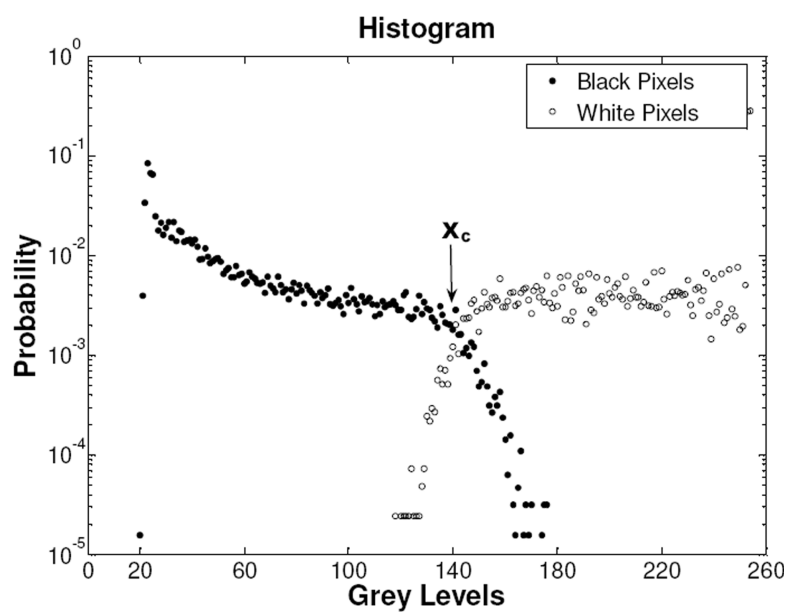

Fig. 2. Distribution of the probability of black and white pixels. 
possible, the exposure is gradually increased as the data pages are stored so that the last data pages have the same BER as the first ones.

In Fig. $\underline{3}$, the exposure time that the data pages have been stored is represented by empty triangles. Thus, when the $300 \times 300$ pixel object was stored, 15 holograms were multiplexed in the material. Figure 3 shows the BER of the four multiplexed data pages as a function of the number of holograms. The BERs obtained from each of the 15 holograms are represented by solid black circles. As can be seen, the first 9 holograms have a BER less than 0.1, and the holograms up to number 12, less than 0.2. However, the BER of the last holograms increases and reaches 0.4 for hologram 15. For the last holograms, no matter how much the exposure was increased, images were obtained with little contrast between pixels, thus indicating that this is the limit for storing images with this thickness and this number of pixels.

Figure 4(a) shows the image of the first hologram, and Fig. $4(\mathrm{~b})$ shows the image of the last hologram with a lower BER (hologram 12), reconstructed with the reference beam. In Fig. 4(a) there is a greater contrast between the white and black pixels, and the edges are well defined. Figure 4(b) has lower contrast than Fig. 4(a), but the pixels are clearly distinguishing ones to others. The last hologram is not represented because it was not well formed.

In the following step, holograms with $400 \times 400$ pixels were multiplexed and 16 holograms were stored. In Fig. 3 the BERs of these 16 holograms are represented by empty circles. As can be seen again, the first holograms have a BER close to 0, whereas from hologram 13 on, the BERs begin to increase. Figures 4(c) and $4(\mathrm{~d})$ show the first and last hologram with a lower BER(hologram 16) of the $400 \times 400$ pixel object. It can be seen that the quality of the stored image changes when the value of BER varies. And finally, holograms corresponding to the $500 \times 500$ pixel (solid squares) and $800 \times 600$ pixel (empty squares) objects were multiplexed. In these two cases, 19 and 20 holograms

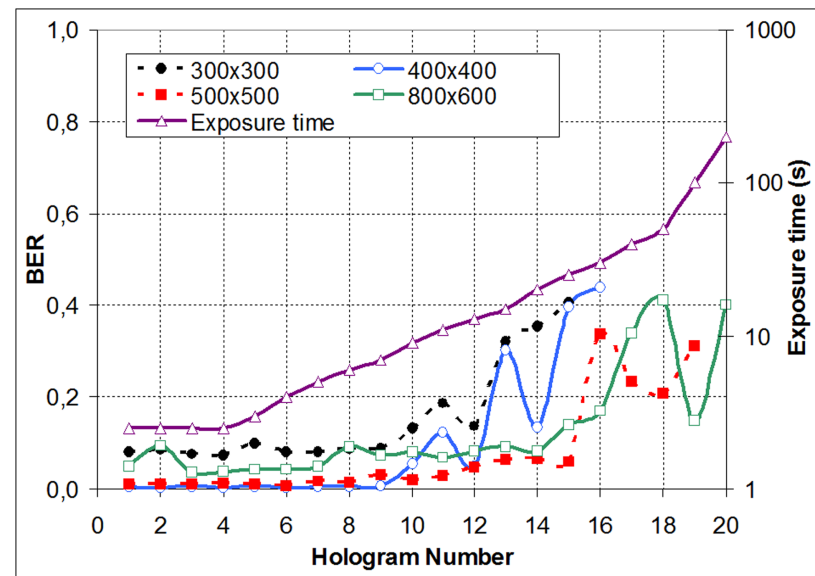

Fig. 3. (Color online) (Color online) BER of the objects with $300 \times$ 300 pixels (solid circles), $400 \times 400$ pixels (empty circles), $500 \times 500$ pixels (solid squares), $800 \times 800$ pixels (empty squares), and exposure time (empty triangles) in a $80 \mu \mathrm{m}$ thick material.

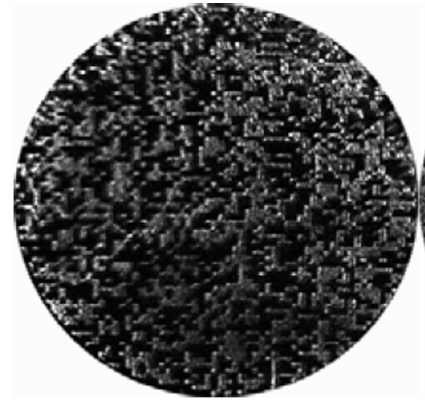

(a)

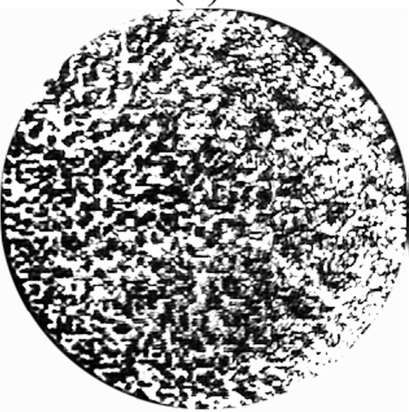

(c)

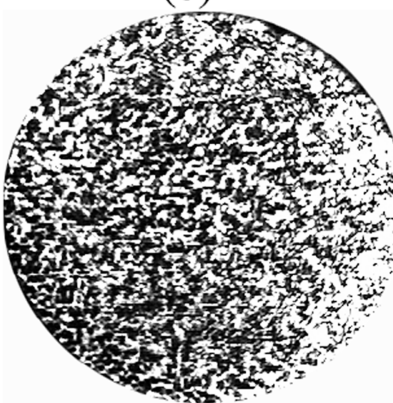

(e)

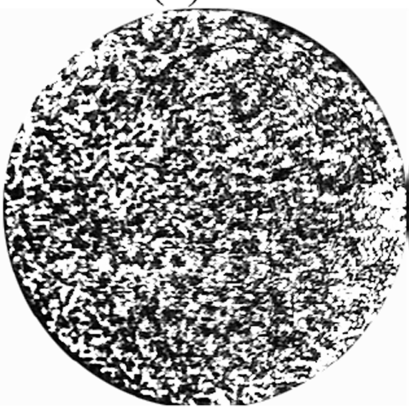

(g)

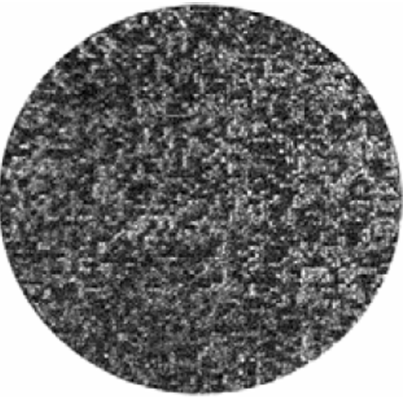

(b)

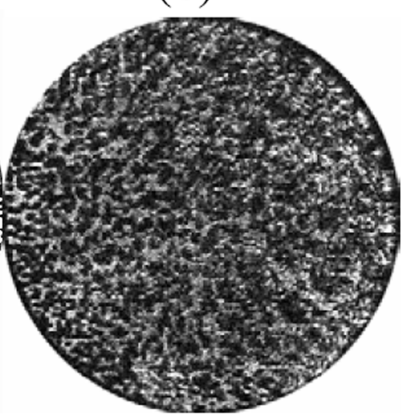

(d)

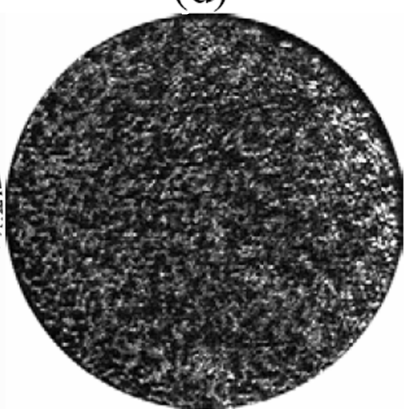

(f)

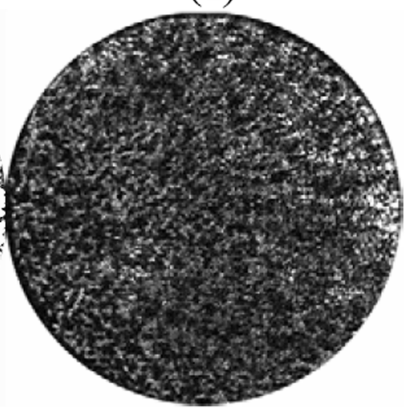

(h)
Fig. 4. First and last holograms with a lower BER corresponding to objects with $300 \times 300$ pixels (a), (b), $400 \times 400$ pixels (c), (d), $500 \times$ 500 pixels (e), (f), and $800 \times 600$ pixels (g), (h) in a $80 \mu \mathrm{m}$ thick layer.

were stored, respectively. As can be seen, the first 15 holograms have a BER of less than 0.2 , and the BER of the last holograms increases but never exceeds 0.4. Figure 4(e) to 4(h) shows the first and last hologram with a lower BER (holograms 15 and 16, respectively) of these two objects. Since the BER of the last data pages stored is higher than that of the others, in future studies a more complete exposure scheduling should be followed to obtain a uniform BER in the reconstructed images. 


\section{Thickness of $250 \mu \mathrm{m}$}

In this subsection, the same objects as those in the previous subsection are multiplexed, but now in a $250 \mu \mathrm{m}$ thick material. In Fig. 5 the BER for these four objects stored with this thickness is represented as a function of the number of holograms. The exposure time that the data pages have been stored is represented by empty triangles. The most important change between Fig. 3 and 5 is that in Fig. 5 more holograms have been store $\bar{d}$ (up to 30 holograms) due to the increase in thickness of the photopolymer layer used.

With this thickness it can be seen that the first holograms (up to hologram 6, depending on the object) have a higher BER than those in the central region (holograms up to number 28 for the $800 \times 600$ pixel object). It has been reported that holograms have a high BER due to the lack of exposure used when storing them. This means that the holograms are not properly formed, and the images obtained are not of good quality. Therefore in these first holograms in which the storage limit has not been reached, having a BER of over 0.1 means that the exposure used to store them was not sufficient. Since the material is thicker, its energetic sensitivity is different, which makes it necessary to use a greater exposure for these first holograms to be formed properly. However, the exposure with which the first two or three holograms should be stored is very critical when greater thicknesses are used $[12,13]$. If the exposure is not sufficient, the hologram is not formed, whereas if the exposure is increased too much, a large part of the dynamic range is consumed and fewer holograms may be stored. For this reason, it is necessary to reach a compromise to obtain the best results. In this study, we opted for sacrificing the first holograms, which meant that they had a slightly higher BER than the central holograms (where the material behaves in a more linear way), in order to be able to store a larger number of holograms. The last holograms again have a high BER since the storage limit for this

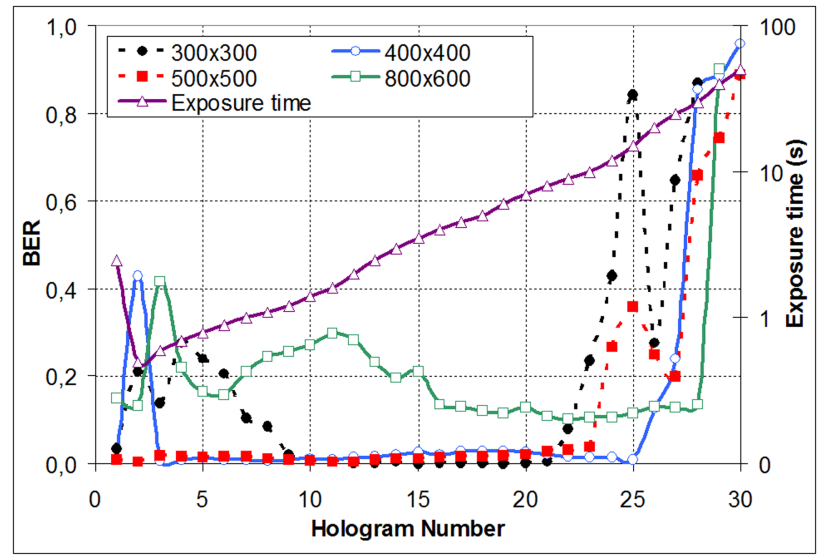

Fig. 5. (Color online) (Color online) BER of the objects with $300 \times$ 300 pixels (solid circles), $400 \times 400$ pixels (empty circles), $500 \times 500$ pixels (solid squares), $800 \times 600$ pixels (empty squares) and exposure time (empty triangles) in a $250 \mu \mathrm{m}$ thick material. thickness has been reached. With the exception of the first and last holograms, it can be seen that the BER of the central holograms is constant and less than 0.1 for the first three objects. However, for the $800 \times 600$ pixel object, the mean BER of the first 25 holograms is greater than that of the other objects.

In Fig. 6 the first and last holograms with a lower BER (holograms 22, 25, 23, and 28 for the objects

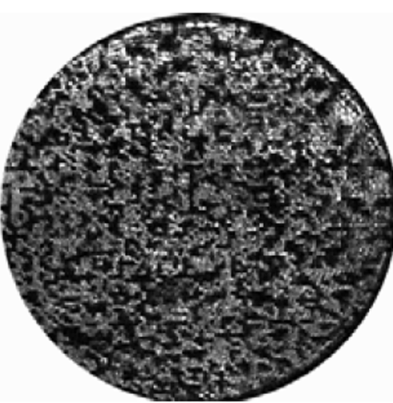

(a)

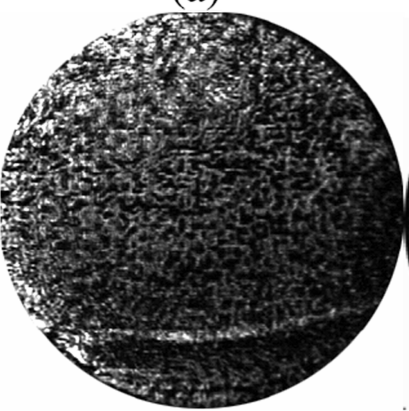

(c)

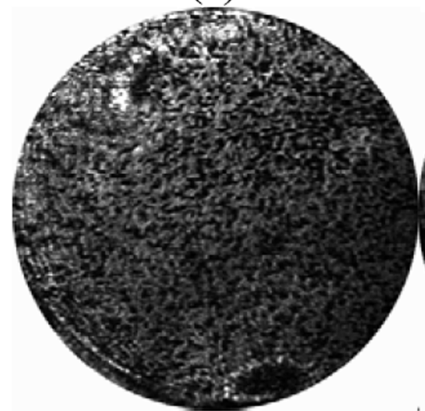

(e)

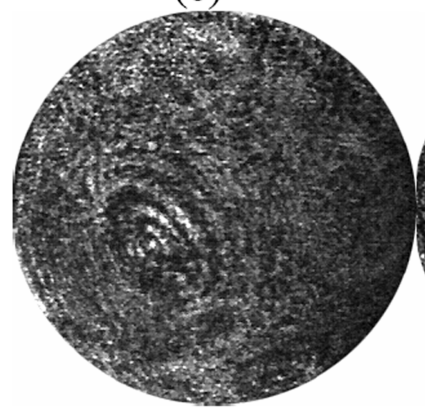

(g)

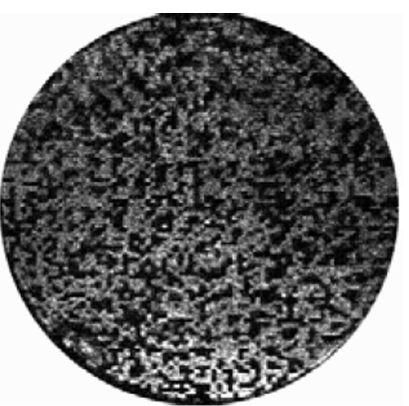

(b)

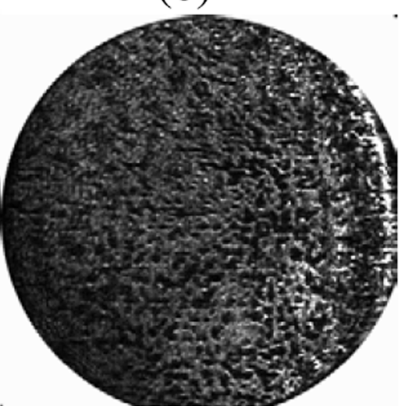

(d)

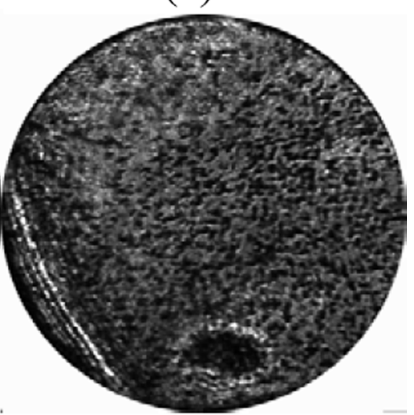

(f)

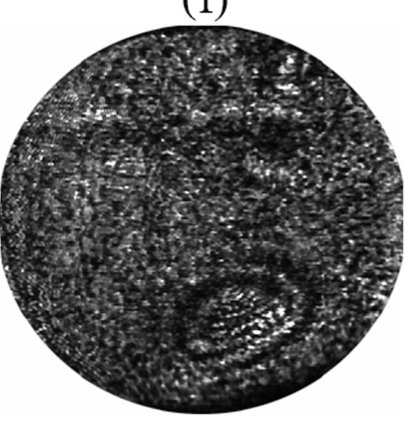

(h)
Fig. 6. First and last holograms with a lower BER corresponding to objects with $300 \times 300$ pixels (a), (b), $400 \times 400$ pixels (c), (d), $500 \times 500$ pixels (e), (f), and $800 \times 600$ pixels (g), (h) in a $250 \mu \mathrm{m}$ thick layer. 
with $300 \times 300$ pixels, $400 \times 400$ pixels, $500 \times 500$ pixels, and $800 \times 600$ pixels, respectively) of the four objects stored in the $250 \mu \mathrm{m}$ thick material are shown. Also, we have included some of the samples with a defect in order to emphasize that, when working with this type of material, and since it is made by us in our laboratory, it is necessary to be very careful because any imperfection or particle deposited may subsequently affect the storage of information. Another reason why we did not reject the plates with a defect is that we believe it is more rigorous to compare the results with plates produced in the same conditions, since sometimes the ambient conditions may vary between one production batch and another, which could affect the experimental results.

In Fig. 6(g) it can be seen that, together with the black and white pixels, a series of diffraction rings have been stored in the center of the image. The material in this region has a small impurity, and this makes rings appear in the reconstruction. This noise due to the impurity is the cause of the increase in the BER. Therefore in order to prevent this type of noise, it is necessary to be very careful when manufacturing the material to ensure there are no small impurities that may cause this undesirable noise in the image.

\section{E. Thickness of $500 \mu \mathrm{m}$}

Finally, the same four objects are multiplexed in the $500 \mu \mathrm{m}$ thick material. Figure 7 shows the BER for these four objects and for the thickness as a function of the number of holograms stored and the exposure time represented by empty triangles. In this case, since the thickness is increased, the dynamic range of the material also increases, thereby enabling a greater number of holograms to be stored. The number of holograms stored ranges from 34 with the $400 \times 400$ pixel object to 70 with the $800 \times 600$ pixel object. As in the case of a thickness of $250 \mu \mathrm{m}$, the first and last holograms were stored with a higher BER than the central holograms. These central holograms have a BER of less than 0.1. In Fig. $\underline{8}$ the first

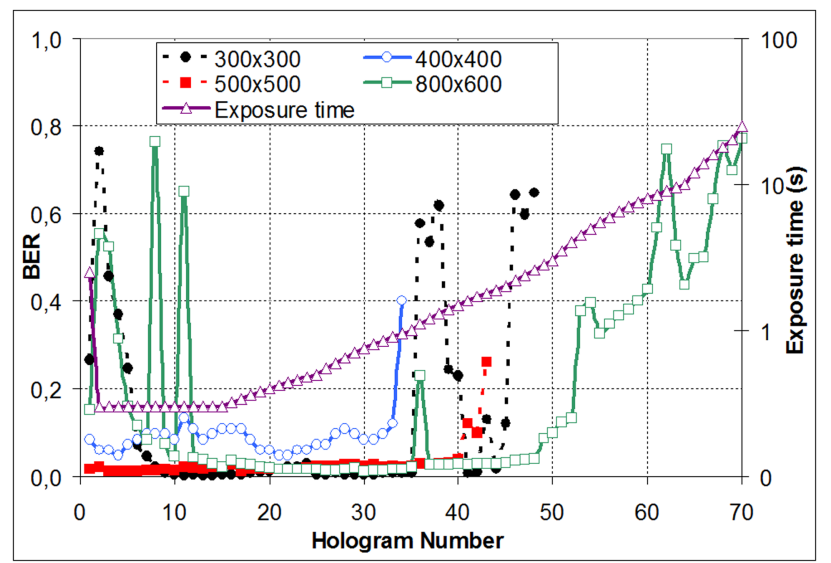

Fig. 7. (Color online) (Color online) BER of the objects with $300 \times$ 300 pixels (solid circles), $400 \times 400$ pixels (empty circles), $500 \times 500$ pixels (solid squares), $800 \times 600$ pixels (empty squares), and exposure time (empty triangles), in a $500 \mu \mathrm{m}$ thick material. and last hologram with a lower BER of the four objects (holograms 35, 33, 40, and 52, respectively) stored in the $500 \mu \mathrm{m}$ thick material are shown.

\section{Conclusion}

The capacity of a PVA/acrylamide photopolymer to multiplex data pages has been demonstrated. For the first time, the images multiplexed in this

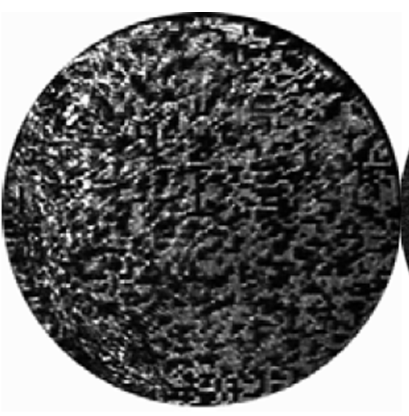

(a)

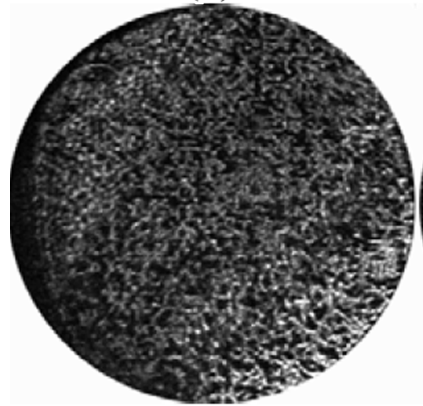

(c)

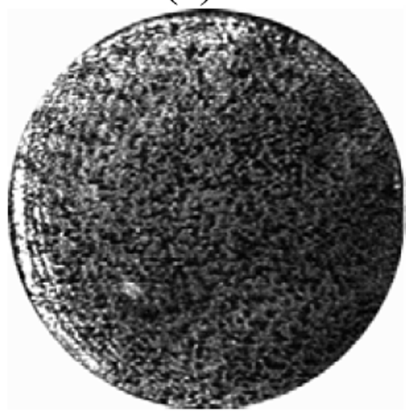

(e)

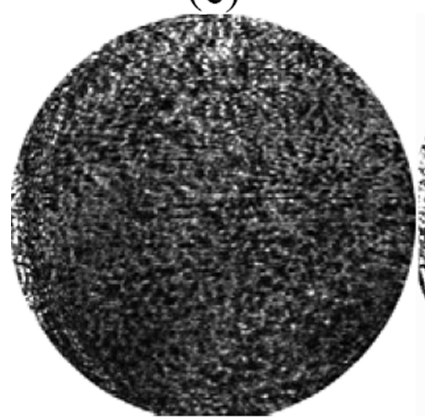

(g)

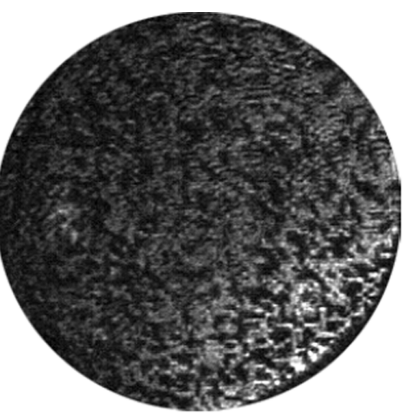

(b)

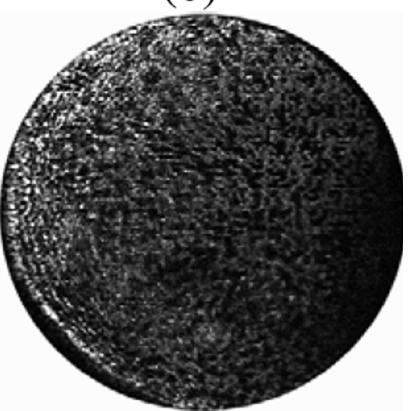

(d)

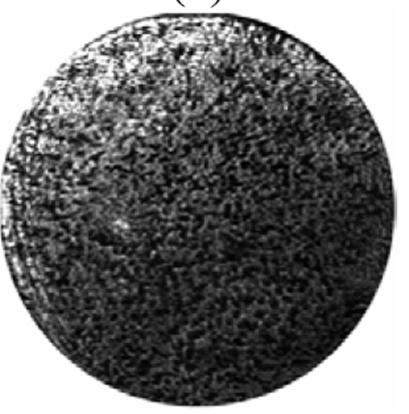

(f)

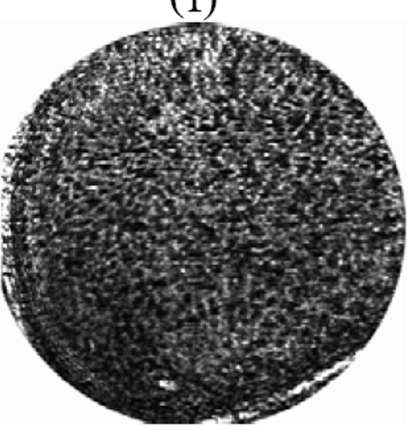

(h)
Fig. 8. First and last holograms with a lower BER corresponding to objects with $300 \times 300$ pixels (a), (b), $400 \times 400$ pixels (c), (d), $500 \times 500$ pixels (e), (f), and $800 \times 600$ pixels (g), (h) in a $500 \mu \mathrm{m}$ thick layer. 
material have been shown. In this study, four different data pages with a different pixel size were multiplexed in a PVA/acrylamide photopolymer. TN-LCDs were used as a spatial light modulator to modify the object beam and store the data pages in the material. The data pages were stored in three layers with different thicknesses. The BER of the multiplexed images was calculated to quantify the quality of the images and represented versus the number of multiplexed holograms.

From the experiments, we conclude that the thickness of the material determines the number of holograms that may be stored, since, when the thickness of the material increases, so does the number of holograms stored. With a thickness of $80 \mu \mathrm{m}$, although the number of holograms stored is less than that for other thicknesses, the first 12 holograms all have the same BER, irrespective of the number of pixels of the object. With thicknesses of $250 \mu \mathrm{m}$ and $500 \mu \mathrm{m}$, the first and last holograms stored have a greater BER. This means that the most appropriate exposure as a function of the type of thickness used should be chosen, or the first images stored rejected. As occurs in the case of multiplexing diffraction gratings, when an exposure scheduling must be chosen in order to obtain uniform diffraction efficiency [30], when multiplexing data pages, an exposure scheduling that gives a uniform BER in the stored images could be used. Nevertheless, it should be noted that there are some 15 central holograms (thickness of $250 \mu \mathrm{m}$ ) and 25 central holograms (thickness of $500 \mu \mathrm{m}$ ) whose BER is less than 0.1, which indicates that the image obtained is of very good quality. This study shows the possibilities of this photopolymer as a holographic memory.

This work was supported by the Ministerio de Educación y Ciencia (Spain) under projects FIS 200505881-C02-01 and FIS 2005-05881-C02-02, by the Ministerio de Ciencia e Innovación under projects FIS 2008-05856-C02-01 and FIS 2008-05856-C0202 , and by Generalitat Valenciana under project GV06/172.

\section{References}

1. A. Pu and D. Psaltis, "High-density recording in photopolymer based holographic three-dimensional disks," Appl. Opt. 35, 2389-2398 (1996).

2. M. Schnoes, B. Ihas, A. Hirl, L. Dhar, D. Michaels, S. Setthachayanon, G. Schomberger, and W. L. Wilson, "Holographic storage media for practical systems," Proc. SPIE 5005, 29-37 (2003).

3. R. R. McLeod, A. J. Daiber, M. E. McDonald, T. L. Robertson, T. Slagle, S. L. Sochava, and L. Hesselink, "Microholographic optical disk data storage," Appl. Opt. 44, 3197-3207 (2005).

4. S. Orlov, W. Phillips, E. Bjornson, Y. Takashima, P. Sundaram, L. Hesselink, R. Okas, D. Kwan, and R. Snyder, "Hightransfer-rate high-capacity holographic disk data-storage system," Appl. Opt. 43, 4902-4914 (2004).

5. S. Gallego, M. Ortuño, C. Neipp, A. Márquez, A. Beléndez, E. Fernández, and I. Pascual, "Three-dimensional characterization of thick grating formation in PVA/AA based photopolymer," Opt. Express 14, 5121-5128 (2006).
6. R. K. Banyal and R. Prasad, "Holographic recording in Fe : $\mathrm{Ce}$ : Ti doped $\mathrm{LiNbO}_{3}$ crystal," Opt. Commun. 274, 300-306 (2007).

7. D. A. Waldman, C. J. Butler, and D. H. Raguin, "CROP holographic storage media for optical data storage greater than $100 \mathrm{bits} / \mu \mathrm{m}^{2}$, Proc SPIE 5216, 10-25 (2003).

8. W. L. Wilson, K. R. Curtis, K. E. Anderson, M. C. Tankitt, A. J. Hirl, M. Pane, C. Stanhope, T. Earhart, W. Loechel, C. Bergman, K. Wolfgang, C. Shuman, G. Hertrich, K. Pharris, K. Malang, and M. Ayres, "Realization of high-performance holographic data storage: the InPhase Technologies demonstration platform," Proc. SPIE 5216, 178-191 (2003).

9. O. Graydon, "Holographic storage turns blue," Opt. Laser Europe 125, 7 (2005).

10. S. Gallego, M. Ortuño, C. Garcia, C. Neipp, A. Belendez, and I. Pascual, "High-efficiency volume holograms recording on acrylamide and N,N'methylene-bis-acrylamide photopolymer with pulsed laser," J. Mod. Opt. 52, 1575-1584 (2005).

11. S. Gallego, M. Ortuño, C. Neipp, A. Márquez, A. Bélendez, and I. Pascual, "Characterization of polyvinyl alcohol acrylamide holographic memories with a first-harmonic diffusion model," Appl. Opt. 44, 6205-6210 (2005).

12. E. Fernández, C. García, M. Ortuño, S. Gallego, A. Beléndez, and I. Pascual, "Optimization of a thick polyvinyl alcoholacrylamide photopolymer for data storage using a combination of angular and peristrophic holographic multiplexing," Appl. Opt. 45, 7661-7666 (2006).

13. E. Fernández, M. Ortuño, S. Gallego, C. García, A. Beléndez, and I. Pascual, "Comparison of peristrophic multiplexing and a combination of angular and peristrophic holographic multiplexing in a thick PVA/acrylamide photopolymer for data storage," Appl. Opt. 46, 5368-5373 (2007).

14. K. Y. Hsu, S. H. Lin, and Y.-N. Hsiao, "Experimental characterization of phenanthrenequinode-doped poly(methyl methacrylate) photopolymer for volume holographic storage," Opt. Eng. 42, 1390-1396 (2003).

15. M. Ortuño, S. Gallego, C. García, C. Neipp, A. Beléndez, and I. Pascual, "Optimization of a $1 \mathrm{~mm}$ thick PVA/acrylamide recording material to obtain holographic memories: method of preparation and holographic properties," Appl. Phys. B 76, 851-857 (2003).

16. M. Ortuño, E. Fernández, A. Márquez, S. Gallego, C. Neipp, A. Beléndez, and I. Pascual, "Effect of the incorporation of $\mathrm{N}, \mathrm{N}^{\prime}$ methylene-bis-acrylamide on the multiplexing of holograms in a hydrophilic acrylamide photopolymer," Opt. Commun. 268, 133-137 (2006).

17. K. Lu and B. E. A. Saleh, "Theory and design of the liquidcrystal TV as an optical spatial phase modulator," Opt. Eng. 29, 240-246 (1990).

18. M. Yamauchi and T. Eiju, "Optimization of twisted-nematic liquid-crystal panels for spatial light phase modulation," Opt. Commun. 115, 19-25 (1995).

19. A. Marquez, C. Iemmi, I. Moreno, J. A. Davis, J. Campos, and M. J. Yzuel, "Quantitative prediction of the modulation behavior of twister-nematic liquid-crystal displays based on a simple physical model," Opt. Eng. 40, 2558-2564 (2001).

20. A. Márquez, J. Campos, M. J. Yzuel, I. Moreno, J. A. Davis, C. Iemmi, A. Moreno, and A. Robert, "Characterization of edge effects in twisted nematic liquid crystal displays," Opt. Eng. 39, 3301-3307 (2000).

21. H. Kim and Y. H. Lee, "Unique measurement of the parameters of a twisted-nematic liquid-crystal display," Appl. Opt. 44, 1642-1649 (2005).

22. V. Duran, J. Lancis, E. Tajahuerce, and Z. Jaroszewicz, "Cell parameter determination of a twisted-nematic liquid-crystal display by single-wavelength polarimetry," J. Appl. Phys. 97, 043101/1-043101/6 (2005). 
23. J. Jang and D. Shin, "Optical representation of binary data based on both intensity and phase modulation with a twisted-nematic liquid-crystal display for holographic digital data storage," Opt. Lett. 26, 1797-1799 (2001).

24. M. R. Gleeson, J. V. Kelly, C. E. Close, F. T. O'Neill, and J. T. Sheridan, "Effects of absorption and inhibition during grating formation in photopolymer materials," J. Opt. Soc. Am. B 23, 2079-2088 (2006).

25. E. Fernández, M. Ortuño, A. Márquez, S. Gallego, A. Beléndez, and I. Pascual, "Optimization of a holographic memory using an LCD and a PVA based photopolymer," Proc. SPIE 6587, 65870J/1-65870J/9 (2007).

26. H. J. Coufal, D. Psaltis, and G. T. Sincerbox, Holographic Data Storage (Springer-Verlag, 2000).
27. L. Dhar, K. Curtis, M. Tackitt, M. Schilling, S. Campbell, W. Wilson, A. Hill, C. Boyd, N. Levinos, and A. Harris, "Holographic storage of multiple high-capacity digital data pages in thick photopolymer systems," Opt. Lett. 23, 17101712 (1998).

28. M. Keskinoz and B. V. K. Vijaya Kumar, "Application of linear minimum mean-squared-error equalization for volume holographic data storage," Appl. Opt. 38, 4387-4393 (1999).

29. P. Varhegyi, P. Koppa, F. Ujhelyi, and Lorincz, "System modeling and optimization of Fourier holographic memory," Appl. Opt. 44, 3024-3031 (2005).

30. A. Pu, K. Curtis, and D. Psaltis, "Exposure schedule for multiplexing holograms in photopolymer films," Opt. Eng. 35, 2824-2829 (1996). 